\title{
A versatile toolkit for high throughput functional genomics with Trichoderma reesei
}

\author{
André Schuster ${ }^{1,2}$, Kenneth S Bruno ${ }^{1}$, James R Collett ${ }^{1}$, Scott E Baker ${ }^{1}$, Bernhard Seiboth² ${ }^{2}$ Christian P Kubicek $^{2^{*}}$ \\ and Monika Schmoll
}

\begin{abstract}
Background: The ascomycete fungus, Trichoderma reesei (anamorph of Hypocrea jecorina), represents a biotechnological workhorse and is currently one of the most proficient cellulase producers. While strain improvement was traditionally accomplished by random mutagenesis, a detailed understanding of cellulase regulation can only be gained using recombinant technologies.
\end{abstract}

Results: Aiming at high efficiency and high throughput methods, we present here a construction kit for gene knock out in $T$. reesei. We provide a primer database for gene deletion using the pyr4, amdS and hph selection markers. For high throughput generation of gene knock outs, we constructed vectors using yeast mediated recombination and then transformed a T. reesei strain deficient in non-homologous end joining (NHEJ) by spore electroporation. This NHEJ-defect was subsequently removed by crossing of mutants with a sexually competent strain derived from the parental strain, QM9414.

Conclusions: Using this strategy and the materials provided, high throughput gene deletion in T. reesei becomes feasible. Moreover, with the application of sexual development, the NHEJ-defect can be removed efficiently and without the need for additional selection markers. The same advantages apply for the construction of multiple mutants by crossing of strains with different gene deletions, which is now possible with considerably less hands-on time and minimal screening effort compared to a transformation approach. Consequently this toolkit can considerably boost research towards efficient exploitation of the resources of $T$. reesei for cellulase expression and hence second generation biofuel production.

Keywords: Trichoderma reesei; Hypocrea jecorina, transformation, vector construction, gene knock-out library, sexual crossing

\section{Background}

The increasing awareness of the limited availability of fossil fuels along with the environmental problems caused by their application initiated considerable research efforts towards clean and sustainable biofuels [1-3]. Thereby, the cellulases required to degrade cellulosic plant materials into small building blocks, which can be metabolized by yeast or other microbes to ethanol or hydrocarbon biofuel precursors, respectively, are one major focus of investigation [4]. Trichoderma reesei (Hypocrea jecorina) is currently the most efficient

\footnotetext{
* Correspondence: ckubicek@mail.zserv.tuwien.ac.at

${ }^{2}$ Research Area of Gene Technology and Applied Biochemistry, Institute for Chemical Engineering, Vienna University of Technology, Gumpendorfer Strasse 1a/1665, A-1060 Wien, Austria

Full list of author information is available at the end of the article
}

producer of enzyme mixtures for degradation of plant materials [5]. The cellulases produced by this fungus are utilized for diverse industrial processes, from biobleaching of textiles, paper recycling to juice extraction and even as additives in animal feeds [6-8].

The long-standing use of cellulase production by $T$. reesei is paralleled by a thorough investigation of the cellulolytic enzyme system of this fungus and its regulation $[9,10]$. With publication of the genomic sequence of $T$. reesei [11], the progress in understanding the mechanisms of cellulase regulation was accelerated. Analysis of the genome indicated, that despite its production efficiency, $T$. reese $i$ has the lowest amount of cellulolytic enzymes among Sordariomycetes at its disposal.

\section{Biomed Central}


In order to gain an understanding of gene regulation, manipulation of the genome of $T$. reesei is indispensible. A transformation system for this fungus has been available for decades with amdS [12], pyr4 [13] and hph [14] being the most frequently used selection marker systems. Also pyrithiamine resistence [15], benomyl resistence [16] and hexokinase [17] have proven useful for transformation of $T$. reesei. Additionally, vector systems enabling excision of the marker gene cassette has facilitated multiple sequential genome modifications despite the limited availability of marker systems $[18,19]$. Nevertheless, the typical efficiency of homologous integration is less than $10 \%$ using these methods.

One of the most important advancements in recent years, for improving the performance of research with T. reesei was the development of strains deficient in non-homologous endjoining (NHEJ) [19,20]. These strains strongly enhance the probability of homologous integration of DNA constructs for deletion or modification of genes. Up to $95 \%$ of transformants were the result of homologous integration events [20]. While in most fungi little discernible phenotype is reported for this mutation, the respective strains are more sensitive to DNA damage [21], the HOG-MAPkinase pathway is up-regulated [22] and effects on genes involved in carbohydrate transport [23] have been observed in some organisms. The major drawbacks of using strains deficient in non-homologous end joining, however, is that this mutation causes telomere shortening and defects in DNA repair [21], both of which negatively influence genome stability and fitness of the respective strains.

Recently, a further tool for work with $T$. reesei became available: After decades of research leading to the conclusion that $T$. reese $i$ is a clonal, asexual derivative of a previously sexual species [24], the capability of this fungus for sexual development was discovered [25]. Besides the physiological relevance, this finding also opens up a wide array of new possibilities for research with $T$. reesei. Crossing can now be used for strain improvement and classical genetics. However, the fact that the parental strain of all $T$. reesei strains used in research and industry, QM6a, is female sterile [25], necessitates the use of a sexually competent wildtype isolate for crossing. Consequently, the genetic background introduced by this closely related but also phenotypically different isolate represents a serious drawback for use of sexual crossing in research.

Genome sequencing and high throughput analysis methods for transcriptomics, proteomics and metabolomics have had considerable impact on how research with fungi progresses [26]. Despite considerable improvements in transformation techniques, the recent increase in available genomic sequences of fungi also caused the need to efficiently use these resources.
However, despite the wealth of data created, in depth functional analysis of genes often lags behind and consequently many fungal genome databases still remain with their most precious treasures undiscovered. Mostly, this is due to the enormous effort necessary for creation of a gene knock out library, which necessitates thousands of experiments and subsequent screenings. Therefore the respective methods need to be streamlined and automated as much as possible. For the model organism, Neurospora crassa, a community-wide effort recently led to the completion of a whole genome knock-out library [27]. This resource is especially important for evaluation of transcriptome data, because screening of a large number of mutant strains considerably increases the knowledge and understanding to be gained from these studies $[28,29]$. Additionally, the possibility to study a group of functionally related genes, such as transcription factors can provide intriguing insights into previously unexplored physiological processes [30,31].

While elaborate molecular biological tools are available for other fungi, especially $N$. crassa, tools for efficient systematic analysis of gene function are relatively under-developed in $T$. reesei. Despite its widespread use in industry, no comprehensive gene knock out library is available for this fungus, nor are there currently efforts to start such an initiative. We therefore aimed to provide a dependable and easy to handle toolkit as a first step towards creation of larger sets of gene knock out strains in $T$. reesei.

In this study we explored strategies to enable high throughput gene-knockout along with efficient construction of multiple mutants. We applied yeast based recombination mediated vector construction, transformation by electroporation and subsequently removal of the NHEJ-deficient background by crossing with a sexually competent $T$. reesei strain derived from QM9414. Genome wide primer libraries for knock out vector construction using different marker systems complete this toolkit. These methods can serve as a basis for construction of large scale libraries of knock out strains for systematic functional genomic studies with $T$. reesei.

\section{Results and discussion}

\section{Vector construction by yeast mediated recombination}

The aim of this study was to enhance the efficiency of construction of deletion mutants to enable a high throughput approach for $T$. reesei. We therefore applied the method of yeast mediated recombination for deletion vector construction, which was shown to be highly efficient [30]. In order to test our approach we selected a set of 20 genes encoding proteins with a variety of predicted functions, such as transporters, transcription factors, peptaibol synthetases or G-protein coupled receptors (Table 1 ). The size of the predicted genes 
Table 1 Overview about selected $T$. reesei genes.

\begin{tabular}{|c|c|c|c|c|c|c|}
\hline $\begin{array}{l}\text { protein } \\
\text { ID }\end{array}$ & $\begin{array}{l}\text { gene } \\
\text { name }\end{array}$ & function & $\begin{array}{l}\text { classification/ } \\
\text { group }\end{array}$ & $\begin{array}{l}\text { ORF length } \\
\text { [bp] }\end{array}$ & $\begin{array}{l}\text { protein } \\
\text { size [aa] }\end{array}$ & $\begin{array}{c}\text { successful } \\
\text { transformation method }\end{array}$ \\
\hline 2526 & $p k c 1$ & protein kinase $\mathrm{C}$ & signal transduction & 3888 & 1140 & $P$ \\
\hline 2845 & gna2 & g-protein alpha subunit two & signal transduction & 1510 & 355 & $E$ \\
\hline 3873 & pde1 & hypothetical cyclic AMP phosphodiesterase, class II & signal transduction & 2177 & 526 & $P$ \\
\hline 21505 & gna3 & g-protein alpha subunit three & signal transduction & 1423 & 356 & $\mathrm{E}$ \\
\hline 23171 & & nonribosomal peptide synthetase (NRPS) & $\begin{array}{l}\text { secondary } \\
\text { metabolism }\end{array}$ & 69518 & 20874 & N \\
\hline 28731 & gpr2 & hypothetical GPCR, family 2 & $\begin{array}{l}\text { G-protein coupled } \\
\text { receptor }\end{array}$ & 1258 & 374 & $\mathrm{E}$ \\
\hline 37515 & rid1 & $\begin{array}{l}\text { DNA methylase, required for RIP (GenBank } \\
\text { Accession number: JN227866) }\end{array}$ & $\begin{array}{c}\text { sexual } \\
\text { development }\end{array}$ & 1815 & 553 & $\mathrm{E}$ \\
\hline 56684 & - & sugar transporter & transport & 1758 & 504 & $P$ \\
\hline 58456 & - & Zn2Cys6 transcription factor & transcription factor & 1589 & 445 & $P$ \\
\hline 65315 & - & basic leucine zipper transcription factor & transcription factor & 1046 & 299 & $P$ \\
\hline 70351 & - & Zn2Cys6 transcription factor & transcription factor & 1670 & 480 & $P$ \\
\hline 72004 & - & hypothetical GPCR, family 2, & $\begin{array}{l}\text { G-protein coupled } \\
\text { receptor }\end{array}$ & 1464 & 442 & N \\
\hline 77795 & $\lim 1$ & putative E3 ubiquitin ligase & signal transduction & 2004 & 637 & $\mathrm{E}$ \\
\hline 79202 & - & $\begin{array}{c}\text { hypothetical transmembrane protein, transport } \\
\text { protein }\end{array}$ & transport & 1575 & 401 & $\mathrm{E}$ \\
\hline 79756 & fwd1 & hypothetical F-Box/WD40 repeat protein & circadian rhythm & 2832 & 920 & $P$ \\
\hline 102497 & - & Zn2Cys6 transcription factor & transcription factor & 2328 & 665 & $P$ \\
\hline 102655 & pde2 & hypothetical phosphodiesterase & signal transduction & 2933 & 857 & $P$ \\
\hline 109088 & hpol & heterochromatin regulator & chromatin binding & 1308 & 272 & $P$ \\
\hline 109146 & - & PTH11-GPCR & $\begin{array}{l}\text { G-protein coupled } \\
\text { receptor }\end{array}$ & 1604 & 452 & $P$ \\
\hline 123786 & - & non-ribosomal peptide synthetase & $\begin{array}{l}\text { secondary } \\
\text { metabolism }\end{array}$ & 50787 & 16535 & $P$ \\
\hline
\end{tabular}

Protein ID's were obtained from the T. reesei Database v2.0. (P: protoplast transformation, E: electroporation of $T$. reesei spores, N: not successful)

ranged between around 1000 to 4000 bp with the exception of the peptaibol synthetases which are larger than $50 \mathrm{~kb}$. For amplification of the flanking sequences of these genes, primers were designed with the following properties: $\mathrm{T}_{\mathrm{m}}: 50-60^{\circ} \mathrm{C}$, GC-content $40-60 \%$ and a length of 20 base pairs. We generated 5' flanking sequences and 3' flanking sequences with a size of around 1000 - $1500 \mathrm{bp}$ 's for each gene (Figure 1A, Additional file 1). In case of TR_23171 and TR_123786 (peptaibol synthetases genes with a size $>50.000$ bp's) we chose as the 3' downstream flanking sequence a 1000 - 1500 bp's fragment from within the open reading frame. This selection should result in a deletion of the first 2200 base pairs of the gene and consequently in a non-functional peptaibol synthetase. As the selectable marker for Trichoderma transformation, we used the orotidine-5' -phosphate decarboxylase (pyr4, TR_74020) for transformation of $\Delta t k u 70$ which is also a uridine auxotroph [20]. The pyr4 gene was amplified from $T$. reesei wild-type DNA and used for yeast mediated recombination with the 3 ' and 5' flanking sequences containing 29 bp homologous overhangs into the shuttle vector pRS426 [30]. Total DNA was isolated from the yeast cells and was used as the template for amplification of the deletion cassettes by PCR.

\section{Construction of deletion mutants in an NHEJ-deficient background}

For high throughput gene deletion a high efficiency of homologous recombination is indispensable. Previous deletion of the $t k u 70$ gene in $T$. reesei TU-6, a uridine auxotrophic derivative of QM9414 [13], resulted in a strain deficient in non homologous endjoining resulting in a homologous integration efficiency which is higher than 95\% [20]. For transformation we used both conventional protoplasting [13] and spore electroporation, as indicated with the respective genes (Table 1). Electroporation was found to be less time consuming and easier to handle,

From the resulting transformants we chose five colonies per gene, which considering the high recombination frequency should result in identification of a positive mutant. For screening we used a PCR based mutant screening. For each gene we designed a specific primer which binds outside from the deletion cassette in the flanking regions of the gene (Additional file 1 Figure 


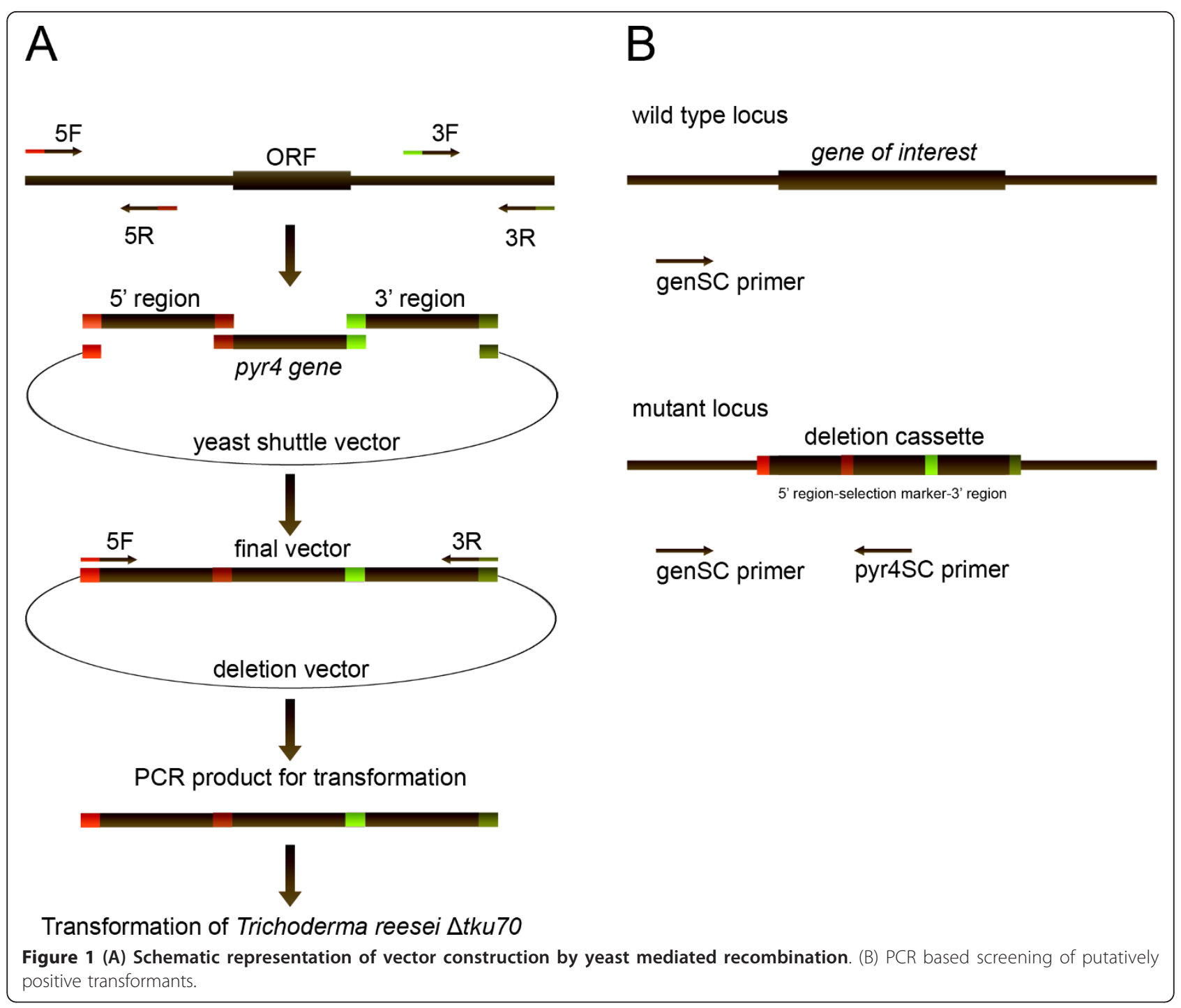

1B). In combination with a specific primer which binds inside of the selectable marker (pyr4) and the primer which binds outside from the cassette we were able to very efficiently screen the mutant strains by PCR. An integration of the deletion cassette at the homologous locus results in a specific amplicon with a size between 1400 to 2000 bp (data not shown) depending on the exact location of the outside primer.

The PCR screening method showed that we were able to generate 18 out of 20 deletion mutant strains. We failed with generation of mutant strains of the following genes: tr_123806, and tr_72004. The frequency of homologous integration was between 33 and 100\%.

\section{Phenotype of Trichoderma reesei mutant strains}

The deletion mutant strains and the control strain ( $\Delta t k u 70$, supplemented with $1 \mathrm{M}$ uridine) were cultivated on malt extract agar $\left(3 \%(\mathrm{w} / \mathrm{v})\right.$ plates at $28^{\circ} \mathrm{C}$ for 6 days. We did not observe severe growth phenotypes for the strains studied, which corresponded well with the available $N$. crassa strains deleted for the predicted orthologous gene as available from the respective genome database http://www.broadinstitute.org/annotation/ genome/neurospora/MultiHome.html (Figure 2).

The mutant lacking NCU00478.5, the homologue of the T. reesei phosphodiesterase gene pde2 (tr_102655), is white due to lack of pigment and no conidia are formed in slants on minimal medium at $25^{\circ} \mathrm{C}$. Similarly, the respective $T$. reesei deletion strain $\Delta p d e 2$ shows reduced growth and white pigmentation. Also the $\Delta f w d 1$ (tr_79756) strain shows reduced and white pigmentation which was also described for its $N$. crassa ortholog (NCU04540.5). Deletion of gpr2 (tr_28731) and hpo1 (tr_109088) in T. reesei leads to a reduced growth on rich medium but no phenotype was described for the orthologous genes (NCU04626.5 and NCU04017.5) 


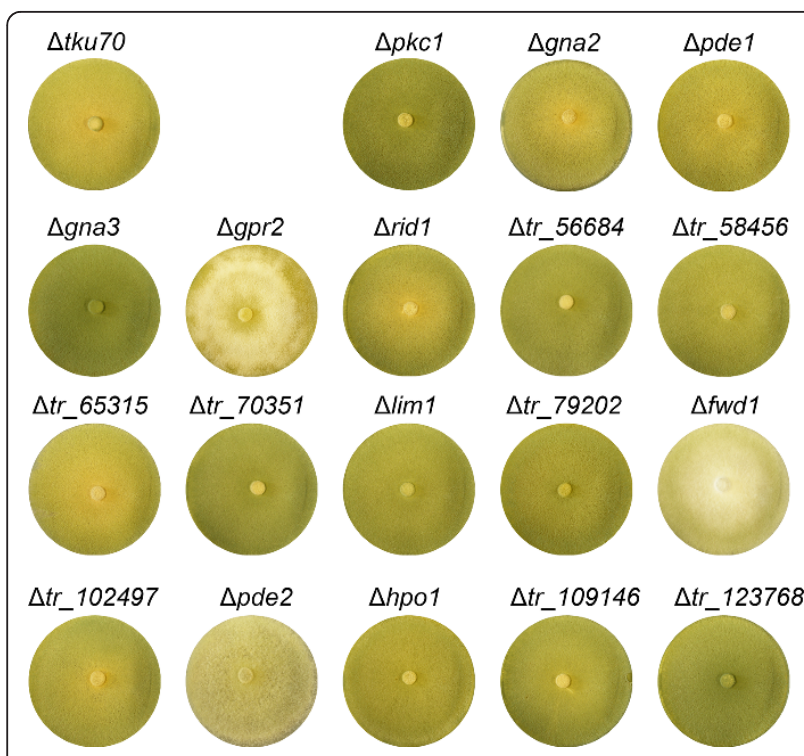

Figure 2 Phenotypes of deletion mutant strains constructed in this study. Strains were grown on malt extract agar plates for six days at $28^{\circ} \mathrm{C}$.

strain in N. crassa. Deletion of gna3 (tr_21505) leads to a higher sporulation on malt extract plates, a phenomenon which was also shown for mutants of this gene in the closely related Trichoderma atroviride [32].

This pilot study showed that using vector construction by yeast mediated recombination, amplification of transformation cassettes and electroporation can significantly reduce hands-on time and considerably enhance efficiency of deletion mutant construction. We therefore went on to design an automated approach for construction of deletion primers for all $T$. reesei genes. This automated approach yielded a full set of primers for 8661 of the 9143 genes in T. reesei v2.0, as well as a partial set of primers for 488 of the genes for use with the pyr4 selection marker system in the NHEJ-deficient background of $T$. reesei $\Delta t k u 70$ (Additional file 2).

\section{Rescue of non homologous end joining by crossing}

Since the deficiency in NHEJ not only brings the advantage of a higher probability of positive transformants, but also the problem of increased sensitivity to DNA damage, decreased genome stability and some changes in gene expression in some fungi [21-23], rescue of wild-type NHEJ is advisable.

The recently described sexual development of $T$. reesei [25] represents a new method for strain development in this fungus. It is possible with this technique to remove undesired mutations by crossing with compatible strains. For this purpose we created a sexually competent strain compatible with our mutants (dubbed QF1; MAT1-1) derived from QM9414 (MAT1-2). Therefore, progeny of a cross between the wild-type isolate Hypocrea jecorina CBS999.97 (MAT1-1) and the female sterile QM9414 were checked for mating type and sexual competence. Strains with MAT1-1 mating type which had gained female fertility were used for further crosses with QM9414. After several rounds of crosses, progeny were checked for desired properties by crossing with CBS999.97 MAT1-1 and CBS999.97 MAT1-2 in order to check for mating type and with QM6a to confirm female fertility (Figure 3A). A female fertile strain of mating type MAT1-1 was selected and checked for its

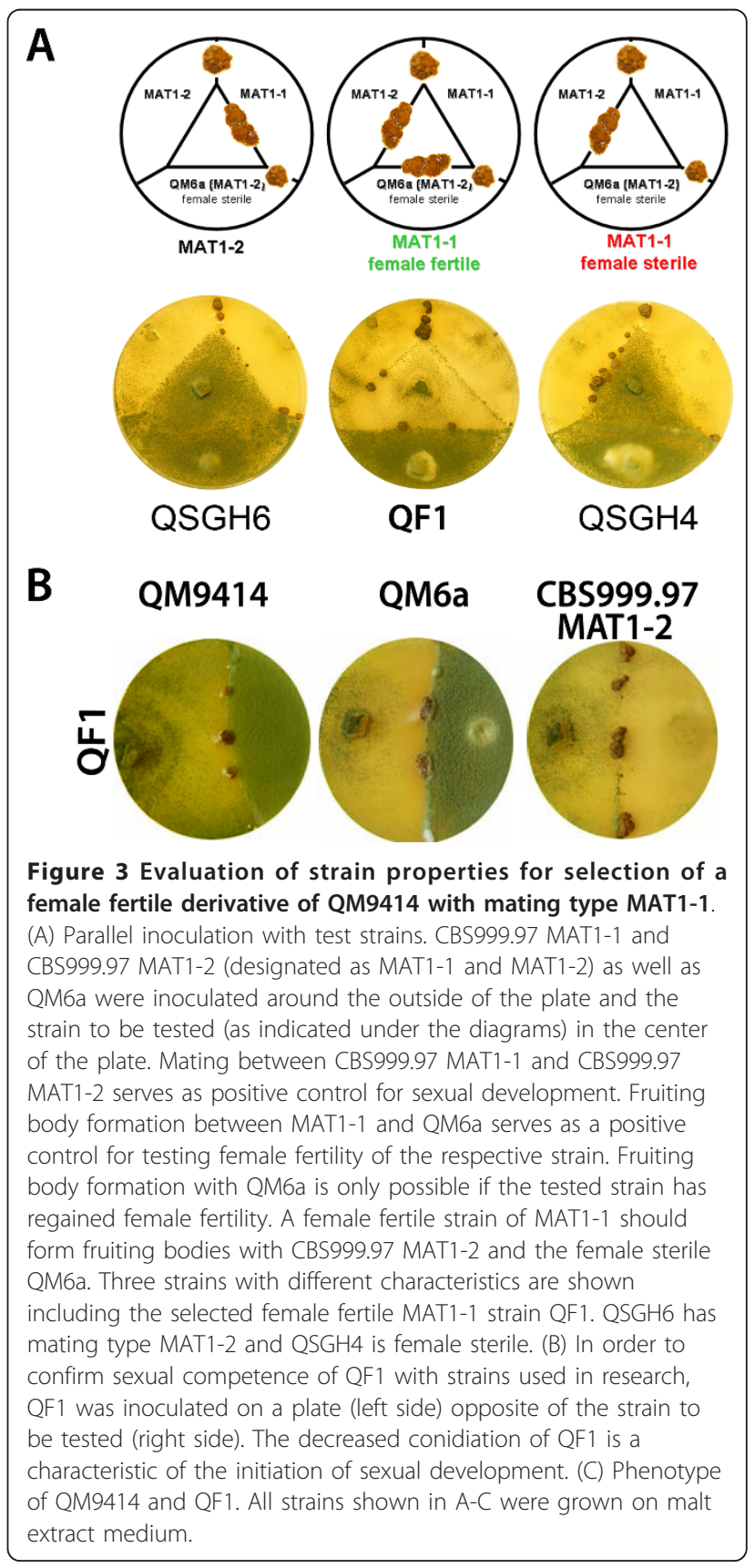


ability to undergo sexual development with QM9414, QM6a and CBS999.97 MAT1-2 (Figure 3B). QF1 shows a similar phenotype as QM9414 as well as comparable cellulase production after five days of growth, albeit also CBS999.97 MAT1-1 produces cellulase levels similar to these strains (data not shown). Consequently we generated a strain with the retained capability of sexual development despite recovery of the QM9414 phenotype.

All mutant strains created in the course of this study are still able to mate with CBS999.97 and also with QF1 despite their deletions. As a proof of principle we selected three deletion strains for the crossing approach aimed at removal of the $t k u 70$ deletion: $\Delta g n a 2, \Delta g n a 3$ and $\Delta$ rid1. While $\Delta g n a 2$ does not show a discernible phenotype, sporulation is increased in $\Delta g n a 3$ and $\Delta$ rid1 is of particular interest, because in this mutant the mechanism of repeat induced point mutation (RIP) is likely to be perturbed. Deletion of the homologue of rid1 (RIP-deficient-1) in N. crassa abolishes RIP [33]. In $T$. reesei this phenomenon has not yet been demonstrated, nevertheless the low frequency of gene duplications in the genome of this fungus could be due to the operation of RIP [11].

Mating assays were performed on malt extract medium [25]. Crosses of these three strains were successful, albeit for $\Delta g n a 3$ lower fertility in sexual crosses was expected [34]. After three weeks the resulting ascospores were isolated randomly and the colonies were grown on selective medium and analyzed. Growth on hygromycin containing medium indicated that the $t k u 70$ deletion was retained, and strains not able to grow in the presence of hygromycin were selected for further analysis. Growth on malt extract medium indicated presence of the initial gene deletion i. e. presence of the functional pyr4 marker gene. Strains growing on malt extract medium without uridine as supplement were checked for presence of the genes of interest. PCR screening confirmed restoration of the wild type $t k u 70$ and showed that the desired gene deletion was retained.

While this method provides a significant improvement compared to retransformation, because no additional marker genes are needed and the hands on time to achieve rescue of the deletion is decreased there are certain issues to be considered. Due to the number of backcrosses, QF1 is estimated to contain approximately 96 - 97\% of the QM9414 genome including the same percentage of QM9414 specific mutations [35]. Thus, QF1 can also be assumed to contain 3 - 4\% of the differences that distinguish QM9414 from CBS999.97.

Interestingly, a recent survey on classical mutations and genome polymorphisms in $N$. crassa [36] revealed significant genomic variation in classical mutant strains in this fungus. Nevertheless, these strains represent the basis for decades of research with $N$. crassa and the estimated level of difference between QF1 and QM9414 is well within the range used historically for Neurospora genetic studies.

The major difference between QF1 and QM9414 is likely to be found in the gene(s) involved in female fertility (and the genes located nearby in the genome), which is functional in QF1 and enables this strain to undergo sexual development with female sterile QM6a and its derivatives including QM9414. The nature of the responsible gene(s) is currently unknown as is its function. Additionally, QF1 has mating type MAT1-1 and hence certain differences in gene expression related to the mating type cannot be excluded [37]. We therefore recommend use of MAT1-2 progeny for further analysis and comparison with QM9414 data.

Although mating is a very efficient means of strain improvement and construction of multiple mutants, the fact that sexual development may introduce alterations in the genome has to be considered. In case of QF1, this includes the unlikely but possible interference of the small portion of the CBS999.97 genome retained in this strain. Therefore, segregation of the observed phenotype of a mutant with the selection marker has to be evaluated for several progeny. Additionally, as required with conventional transformations, different mutant strains have to be checked to confirm the effect of the mutation.

\section{Increasing utility of the system by additional selection markers}

Since the mutation causing uridine-auxotrophy of $\Delta t k u 70$ is not a complete deletion of the pyr4 gene [13], the preconditions for the operation of RIP (homologous sequences longer than $400 \mathrm{bps}$ ) would be fulfilled [38]. Use of alternative selection markers will likely prove to be beneficial. We consequently modified the full genome primer set described above for use with the amdS marker system [12], which can also be used with the NHEJ deficient strain $\Delta t k u 70$ [20] (Additional file 3). Additionally, we provide this primer set for use with the $h p h$ marker cassette (Additional file 4) used in the N. crassa high throughput gene knock out program [30] appropriate for transformation of the NJEH deficient strain QM6a $\Delta$ tmus53 [19]. These marker systems do not involve the use of homologous sequences of $T$. reese $i$ and thus circumvent possible problems with RIP. Alternatively, $\Delta$ rid 1 could be used for these constructions, which however would require subsequent removal of this background in order to avoid side effects by this mutation.

\section{Conclusions}

The study presented here is intended to serve as a basis for fast and efficient analysis of gene function in $T$. 
reesei. Recent studies on genomes of early industrial mutants of $T$. reesei revealed several interesting mutations in these strains $[35,39,40]$, but nevertheless, in many cases the specific genes responsible for the effect on cellulase gene expression as well as possible synergistic effects in these mutant strains, remain to be elucidated. Regulation of cellulase gene expression has been a focus of research with $T$. reesei for decades and hence a considerable number of transcriptome studies are available $[41,42]$. Since the availability of the genome sequence of $T$. reesei [11] several approaches using genome-wide microarrays have been reported $[43,44]$. These studies provide intriguing insights into various aspects of cellulase gene expression, from transcription factors to secretion pathways. Investigation of the target genes identified to be involved in cellulase regulation by analysis of gene knock out strains largely covering the involved pathways would be a logical further step towards elucidation of the mechanisms triggering induction, transcription, processing and secretion of cellulolytic enzymes.

The high relevance of $T$. reesei for the production of economically feasible second generation biofuels from cellulosic agricultural waste instead of food crops still requires considerable improvement of the efficiency of the cellulase mixture secreted [45]. Moreover, the use of T. reesei as a host for heterologous protein production necessitates a more detailed understanding of the physiology of this fungus. Our streamlined system for knockout-construction along with the convenient and easy to use method to create multiple mutants by crossing can serve as a starting point for large scale functional analysis studies aimed to uncover the hidden treasures of the biotechnological workhorse $T$. reesei.

\section{Methods}

\section{Strains and plasmids}

$T$. reesei $\Delta t k u 70$, which is defective in the non-homologous end-joining pathway [20] was used in the present study and was maintained on potato dextrose agar (PDA, Difico, Detroit, USA) which was supplemented with $5 \mathrm{mM}$ uridine (Sigma Aldrich, St. Louis, USA) in order to complement the auxotrophy of strain TU-6, a derivative of QM9414 [13]. Parental strain QM9414 (ATCC 26921; [35,46]) and strains derived from it were maintained on $3 \%(\mathrm{w} / \mathrm{v})$ malt extract with $2 \%(\mathrm{w} / \mathrm{v})$ agar $28^{\circ} \mathrm{C}$. Mating experiments were performed as described previously [25]. Briefly, strains were grown on $3 \%(w / v)$ malt extract medium with $2 \%(\mathrm{w} / \mathrm{v})$ agar. Plates were incubated at $20-25^{\circ} \mathrm{C}$ in daylight for 10 - 14 days until formation of fruiting bodies and ascospore discharge. For yeast transformation the shuttle vector pRS426 [47] and the yeast strain WW-YH10 (ATCC number: 208405) were used.

\section{Vector construction for gene deletion}

For construction of deletion vectors for the selected genes (Table 1) the orotidine-5'-phosphate decarboxylase gene of $T$. reesei (pyr4, TR_74020) was used as selectable marker. The marker gene was amplified using primers pyr4F and pyr4R (supplementary file 1 ). The 50 $\mu \mathrm{l}$ reaction mixture contained $1.25 \mathrm{U}$ Takara Ex Taq ${ }^{\mathrm{TM}}$ (Takara Bio, Madison, Wisconsin), $1 \times$ Ex Taq $^{\text {TM }}$ Buffer, $0.2 \mathrm{mM}$ dNTP, $0.1 \mu \mathrm{M}$ forward and reverse primer, $1 \mu \mathrm{l}$ T. reesei QM9414 genomic DNA $(90 \mathrm{ng} / \mu \mathrm{l})$ as template and nuclease free water. The selectable marker PCR fragment was purified using the E.Z.N.A. Gel Extraction Kit (Omega Bio-Tek, Inc., Norcross, USA).

For generation of the respective 5' and 3' flanking sequences, primers (Table 1) were designed with the OligoExplorer software (version 1.1.2; http://www.genelink.com/tools/gl-oe.asp). In order to enable yeastmediated recombination of the deletion cassette, linker sequences were added to the primers for amplification of the 5' and 3' flanking region (supplementary file 1; Figure 1). Sequences were obtained from: T. reesei genome database (JGI webpage: http://genome.jgi-psf.org/).

The PCR mixture contained 1.25 U Takara Ex Taq ${ }^{\mathrm{TM}}$ (Takara Bio, Madison, Wisconsin), $1 \times$ Ex Taq $^{\mathrm{TM}}$ Buffer, $0.2 \mathrm{mM}$ dNTP, $0.1 \mu \mathrm{M}$ forward and reverse primer, $1 \mu \mathrm{l}$ T. reesei wild-type genomic DNA $(90 \mathrm{ng} / \mu \mathrm{l})$ as template and nuclease free water.

\section{Database generation}

A genome-wide database of primers for the 5' and 3' fragments for construction of the deletion cassette by yeast mediated recombination was generated using a Python script (provided by Peter Andrews of the Neurospora Genome Project) that serialized the function of the Primer3 core primer design program [48]. A masked genome sequence file and a gene annotation file for $\mathrm{T}$. reesei v2.0 were downloaded from the Joint Genome Institute (JGI; http://genome.jgi-psf.org/Trire2/Trire2. download.html) and converted into a format suitable for input into the Python script. The default parameters which were used for Primer3 were the predefined 29 nucleotide sequences attached to the primers (supplementary file 1) and the following settings: optimal primer length $=20 \mathrm{bp}$, minimum primer length $=19 \mathrm{bp}$, flanking region length $=1500 \mathrm{bp}$, primer product size $=$ min: $1000 \mathrm{bp}$ and max.: $1300 \mathrm{bp}$, maximal size of GC clamp in primer $=2$, primer optimal temperature $=56^{\circ}$ $\mathrm{C}$, primer $\mathrm{GC}$ content $=\min : 50 \%$ and $\max : 65 \%$, monovalent catio salt concentration $=50 \mathrm{mM}$ and concentration of annealing DNA oligo $=200 \mathrm{nM}$.

\section{Vector construction and yeast mediated recombination}

The yeast shuttle vector pRS426 was digested with EcoRI and XhoI (restriction enzymes were purchased 
from New England Biolabs, Ontario, Canada) and purified with the E.Z.N.A. Gel Extraction Kit (Omega BioTek, Inc., Norcross, USA). Yeast transformation and preparation was performed essentially as described previously $[30,49,50]$.

An overnight culture $\left(200 \mathrm{rpm}, 30^{\circ} \mathrm{C}\right)$ of the yeast strain WW-YH10 was prepared. $1 \mathrm{ml}$ of the overnight culture was added to $50 \mathrm{ml}$ of fresh YPD (1\% yeast extract, $2 \%$ peptone, $1 \%$ glucose (all chemicals from Sigma Aldrich, St. Louis, USA unless noted otherwise)) medium and incubated at $30^{\circ} \mathrm{C}$ until O.D.600 $=1$. The cells were centrifuged at $14.000 \mathrm{rpm}$ for $3 \mathrm{~min}$. The supernatant was discarded and the pellet was washed with $25 \mathrm{ml}$ of sterile water and centrifuged again for 3 min. The supernatant was discarded and the pellet was resuspended in $400 \mu \mathrm{l}$ of $100 \mathrm{mM}$ lithium acetate (Sigma Aldrich). $50 \mu \mathrm{l}$ of yeast cell suspension was centrifuged for $20 \mathrm{sec}$ and $240 \mu \mathrm{l} 50 \%$ PEG 3550, $36 \mu \mathrm{l} 1 \mathrm{M}$ lithium acetate, $50 \mu \mathrm{l}$ sheared salmon sperm DNA (2 $\mathrm{mg} / \mathrm{ml}$ ) (Sigma Aldrich) and $34 \mu \mathrm{l}$ sterile water was added to the pelleted cells. Thereafter, $200 \mathrm{ng}$ of 5' DNA fragment, $200 \mathrm{ng}$ of 3' DNA fragment, $100 \mathrm{ng}$ of digested pRS426 and $100 \mathrm{ng}$ of selectable marker fragment was added and mixed. After the heat shock for 30 $\min$ at $42^{\circ} \mathrm{C}$ the mixture was centrifuged for $15 \mathrm{sec}$ at $14.000 \mathrm{~g}$ and the supernatant was discarded. $1 \mathrm{ml}$ of sterile water was added, and after thorough mixing centrifuged for $15 \mathrm{sec}$ again. $800 \mu \mathrm{l}$ of supernatant was discarded and the rest was spread onto SC-URA $(1.54 \mathrm{~g} / \mathrm{l}$ BSM-Iso-Ura Powder, $1.71 \mathrm{~g} / \mathrm{l}$ yeast nitrogen base (Sunrise Science Products, San Diego, USA), 5 g/l ammonium sulfate, $20 \mathrm{~g} / \mathrm{l}$ glucose and $20 \mathrm{~g} / \mathrm{l}$ agar (Sigma Aldrich, St. Louis, USA)) plates and incubated for 3-4 days at $30^{\circ} \mathrm{C}$. The resulting colonies were resuspended in $2 \mathrm{ml}$ of sterile water and transferred into a new reaction tube. The sample was centrifuged for $15 \mathrm{sec}$ at $14.000 \mathrm{~g}$ and the supernatant was discarded. $400 \mu \mathrm{l}$ of smash-and-grab lysis buffer ( $2 \%$ Triton X-100, 1\% SDS, $100 \mathrm{mM} \mathrm{NaCl}, 10 \mathrm{mM}$ Tris pH 8.0, 1 mM EDTA), 500 $\mu \mathrm{l}$ phenol $(\mathrm{pH}=7.8), 500 \mu \mathrm{l}$ chloroform/isoamylalcohol (48:2) and $0.3 \mathrm{~g}$ of glass beads were added and mixed well. After centrifugation for $10 \mathrm{~min}$ at $14.000 \mathrm{~g}$ the supernatant was transferred to a new reaction tube and $500 \mu \mathrm{l}$ of chloroform was added and mixed. The supernatant, after centrifugation for $10 \mathrm{~min}$ at $14.000 \mathrm{~g}$, was transferred to a new reaction tube and $0.3 \mathrm{M}$ sodium acetate (pH 5.2) and 2.5 volumes of ethanol (96\%) were added. After precipitation and centrifugation for $10 \mathrm{~min}$ at $14.000 \mathrm{~g}$, the pellet was washed with $400 \mu \mathrm{l} 70 \%$ ethanol and resuspended in $50 \mu \mathrm{l}$ of sterile water. The final linear deletion cassette was amplified from this solution by PCR. The mixture contained $1.25 \mathrm{U}$ Takara Ex Taq $^{\mathrm{TM}}$ (Takara-Bio, Madison, USA) appropriate buffer as described in the application manual, $0.2 \mathrm{mM}$ dNTP,
$0.1 \mu \mathrm{M}$ forward (5F) and reverse primer (3R), $2 \mu \mathrm{l}$ of isolated yeast DNA (approx. $100 \mathrm{ng}$ ) and nuclease free water. The protocol was: $94^{\circ} \mathrm{C} 3: 00$ min followed by 3 cycles of $94^{\circ} \mathrm{C}, 30 \mathrm{sec} ; 65^{\circ} \mathrm{C}, 30 \mathrm{~s} ; 72^{\circ} \mathrm{C}, 2: 00 \mathrm{~min}$; followed by 2 cycles of $94^{\circ} \mathrm{C}, 30 \mathrm{sec} ; 63^{\circ} \mathrm{C}, 30 \mathrm{~s} ; 72^{\circ} \mathrm{C}, 2: 00$ min; followed by 30 cycles of $94^{\circ} \mathrm{C}, 30 \mathrm{sec} ; 60^{\circ} \mathrm{C}, 30 \mathrm{~s}$; $72^{\circ} \mathrm{C}, 2: 00 \mathrm{~min}$; and a final extension of $72^{\circ} \mathrm{C}, 10: 00 \mathrm{~min}$. The final linear deletion cassette was purified using the E.Z.N.A. Gel Extraction Kit (Omega Bio-Tek, Inc., Norcross, USA).

\section{Transformation of $T$. reesei}

The T. reesei $\Delta t k u 70$ strain [20] was used for transformation in order to achieve a high efficiency in homologous integration of the deletion cassettes. The protoplast transformation was carried out as previously described [13]. For transformation $2.5 \mu \mathrm{g}$ of purified deletion cassette fragment was used. Transformants were grown on selective minimal medium ( $1 \mathrm{~g} /$ liter $\mathrm{MgSO}_{4} * 7 \mathrm{H}_{2} \mathrm{O}, 10 \mathrm{~g} /$ liter $1 \% \mathrm{KH}_{2} \mathrm{PO}_{4}, 6 \mathrm{~g} /$ liter $\left(\mathrm{NH}_{4}\right)_{2} \mathrm{SO}_{4}, 3 \mathrm{~g} /$ liter trisodium citrate $2 \mathrm{H}_{2} \mathrm{O}, 10 \mathrm{~g} /$ liter glucose, $20 \mathrm{ml} /$ liter $50 \mathrm{x}$ trace elements solution $\left(0.25\right.$ g/liter $\mathrm{FeSO}_{4} * 7 \mathrm{H}_{2} \mathrm{O}, 0.07 \mathrm{~g} /$ liter $\mathrm{ZnSO}_{4} * 2 \mathrm{H}_{2} \mathrm{O}, 0.1 \mathrm{~g} /$ liter $\mathrm{CoCl}_{2} * 6 \mathrm{H}_{2} \mathrm{O}, 0.085 \mathrm{~g} /$ liter $\mathrm{MnSO}_{4}{ }^{*} \mathrm{H} 2 \mathrm{O}$ ), $2 \%$ (wt/vol) agar; all chemicals were from Sigma Aldrich, St. Louis, USA).

The protocol for electroporation of $T$. reesei was adapted from patent application US2010/0304468. Spores of T. reesei $\Delta t k u 70$ were harvested from a freshly sporulated $90 \mathrm{~mm}$ malt extract agar plate and suspended in $1.1 \mathrm{M}$ sorbitol. Spores were washed twice, resuspended in $100 \mu \mathrm{l} 1.1 \mathrm{M}$ sorbitol and cooled on ice. $75 \mu \mathrm{l}$ of cold spore suspension was mixed with $10 \mu \mathrm{g}$ of vector of interest. For electroporation we used an Electroporation System ECM ${ }^{\circledR} 630$ (BTX Instrument Division Harvard Apparatus, Holliston, USA). 1.8 kV, $800 \Omega$ and $25 \mu \mathrm{F}$ were used as setting for the $\mathrm{ECM}^{\circledR} 630$ device. Thereafter one volume of the reaction mixture (consisting of spore suspension plus deletion cassette) of YEPD (1\% (w/v) yeast extract, $2 \%(\mathrm{w} / \mathrm{v})$ peptone, $1 \%(\mathrm{w} /$ v) glucose) and four reaction volumes $1.1 \mathrm{M}$ sorbitol were added and mixed. For regeneration the whole mixture was incubated over night at room temperature. Thereafter, spores were streaked out on plates containing selection medium.

Compared to the classical protoplast transformation technique [13] transformation by electroporation is less time consuming, easier to perform and the efficiency of this method was comparable to that of protoplast transformation.

Putative deletion strains were tested for integration of the construct by PCR using primer pyr4Sc (inside in the selectable marker gene pyr4) and the gene-specific primer, geneSc (outside from the transformation cassette) (supplementary file 1). Successful homologous 
integration results in a specific amplicon (1400 - 2000 bp's, depending on the gene) (supplementary file 1) and no amplification was possible with $T$. reesei $\Delta t k u 70$ genomic DNA (data not shown).

\section{Construction of a sexually competent derivative of QM9414}

The female sterile wild type strain QM9414 (MAT 1-2) and the sexually competent wild-type strain CBS999.97 (MAT1-1) [25] were inoculated on $3 \%(\mathrm{w} / \mathrm{v})$ malt extract medium with $2 \%(\mathrm{w} / \mathrm{v})$ agar for two to three weeks at $28^{\circ} \mathrm{C}$ until ascospore discharge. The ascospores were isolated from the lid with sterile water. This solution was spread out on a $3 \%(\mathrm{w} / \mathrm{v})$ malt extract medium with $2 \%(\mathrm{w} / \mathrm{v})$ agar plate and incubated at $28^{\circ} \mathrm{C}$ for two days until single colonies could be observed. The resulting colonies were screened for the mating type and again crossed to QM9414. The number of sexually competent strains decreased after each round of crossing, indicating a small region of the genomic to be relevant for this process. After five rounds of backcrossing, sexually competent strains with mating type MAT1-1 were tested for their phenotype upon growth on plates and a strain with characteristics largely indistinguishable from QM9414 was selected for further analysis.

For comparison of cellulase production, the parental strain QM9414 and QF1 were grown in $200 \mathrm{ml}$ of liquid Mandels-Andreotti minimal medium with $1 \%(\mathrm{w} / \mathrm{v})$ microcrystalline cellulose (No. 14204; Serva, Heidelberg, Germany) as carbon source at $200 \mathrm{rpm}$ and $28^{\circ} \mathrm{C}$ in a rotary shaker. After 5 days of cultivation, samples were harvested and biomass formation and cellulase activity were measured as described previously [51].

\section{Crossing of the deletion mutant strains against QF1}

The deletion strains were crossed with QF1 as described previously [25]. Ascopores were isolated and colonies were grown on selective medium in order to obtain progeny carrying the desired gene deletion, but not the $\Delta t k u 70$ background. The resulting strains were tested for restoration of the $t k u 70$ locus using primers tku70ORFF and tku70ORFR. DNA was isolated with standard protocols. Successful restoration results in a specific band (480 bp's) for $t k u 70$ whereas no amplification was possible in the $\Delta t k u 70$ background. Screening of mutants for propagation of the desired deletion was performed by PCR as described above.

\section{Additional material}

Additional file 1: Oligonucleotides used for vector construction and screening. This file contains primer sequences, the respective fragment sizes of the amplicons prepared for vector construction along with the size of the amplicon for transformant screenings.
Additional file 2: Oligonucleotides for high throughput yeast mediated vector construction using the pyr4 marker system.

Additional file 3: Oligonucleotides for high throughput yeast mediated vector construction using the amdS marker system. Additional file 4: Oligonucleotides for high throughput yeast mediated vector construction using the hph marker system.

\section{Acknowledgements}

We want to thank Kulwant Kandra for technical assistance with selected analyses. Work of AS and MS was supported by grants from the Austrian Science Fund (FWF; P20004 and V152-B20) to MS. The T. reesei genome sequencing project was done at the Joint Genome Institute (JGI) funded by the US Department of Energy.

\section{Author details}

${ }^{1}$ Chemical and Biological Process Development, Energy and Environment Directorate, Pacific Northwest National Laboratory, 902 Battelle Blvd, Richland, WA, USA. ${ }^{2}$ Research Area of Gene Technology and Applied Biochemistry, Institute for Chemical Engineering, Vienna University of Technology, Gumpendorfer Strasse 1a/1665, A-1060 Wien, Austria.

\section{Authors' contributions}

AS performed primer design, vector construction, transformation and screening of knock out strains and wrote the draft version of the manuscript, KSB and BS participated in conception of the study, KSB and and SEB supervised construction of knock out strains, JRC prepared the whole genome primer database, CPK and SEB conceived of the study, MS participated in conception of the study, suggested and constructed the backcrossed strain QF1, supervised work of AS and wrote the final version of the manuscript.

All authors have read and approved the final manuscript.

\section{Competing interests}

The authors declare that they have no competing interests.

Received: 20 September 2011 Accepted: 2 January 2012

Published: 2 January 2012

\section{References}

1. Rubin EM: Genomics of cellulosic biofuels. Nature 2008, 454:841-845.

2. Kubicek CP, Herrera-Estrella A, Seidl-Seiboth V, Martinez DA, Druzhinina IS, Thon M, Zeilinger S, Casas-Flores S, Horwitz BA, Mukherjee PK, et al: Comparative genome sequence analysis underscores mycoparasitism as the ancestral life style of Trichoderma. Genome Biol 2011, 12:R40.

3. Somerville C: Biofuels. Curr Biol 2007, 17.

4. Kumar R, Singh S, Singh OV: Bioconversion of lignocellulosic biomass: biochemical and molecular perspectives. J Ind Microbiol Biotechnol 2008, 35:377-391.

5. Schuster A, Schmoll M: Biology and biotechnology of Trichoderma. Appl Microbiol Biotechnol 2010, 87:787-799.

6. Buchert J, Oksanen T, Pere J, Siika-Aho M, Suurnäkki A, Viikari L: Applications of Trichoderma reesei enzymes in the pulp and paper industry. In Trichoderma and Gliocladium. Edited by: Harman GE, Kubicek CP. London: Taylor and Francis; 1998:343-363.

7. Galante Y, De Conti A, Monteverdi R: Application of Trichoderma enzymes in the textile industry. In Trichoderma and Gliocladium. Volume 2. Edited by: Harman GE, Kubicek CP. London: Taylor and Francis; 1998:311-326.

8. Galante Y, De Conti A, Monteverdi R: Application of Trichoderma enzymes in the food and feed industries. In Trichoderma and Gliocladium. Volume 2. Edited by: Harman GE, Kubicek CP. London: Taylor and Francis; 1998:327-342.

9. Kubicek CP, Mikus M, Schuster A, Schmoll M, Seiboth B: Metabolic engineering strategies for the improvement of cellulase production by Hypocrea jecorina. Biotechnol Biofuels 2009, 2:19.

10. Schmoll M, Kubicek CP: Regulation of Trichoderma cellulase formation: lessons in molecular biology from an industrial fungus. A review. Acta Microbiol Immunol Hung 2003, 50:125-145. 
11. Martinez D, Berka RM, Henrissat B, Saloheimo M, Arvas M, Baker SE, Chapman J, Chertkov O, Coutinho PM, Cullen D, et al: Genome sequencing and analysis of the biomass-degrading fungus Trichoderma reesei (syn. Hypocrea jecorina). Nat Biotechnol 2008, 26:553-560.

12. Penttila M, Nevalainen $H$, Ratto $M$, Salminen $E$, Knowles J: A versatile transformation system for the cellulolytic filamentous fungus Trichoderma reesei. Gene 1987, 61:155-164.

13. Gruber F, Visser J, Kubicek CP, de Graaff LH: The development of a heterologous transformation system for the cellulolytic fungus Trichoderma reesei based on a pyrG-negative mutant strain. Curr Genet 1990, 18:71-76.

14. Mach RL, Schindler M, Kubicek CP: Transformation of Trichoderma reesei based on hygromycin $B$ resistance using homologous expression signals. Curr Genet 1994, 25:567-570.

15. Kubodera T, Yamashita N, Nishimura A: Transformation of Aspergillus sp. and Trichoderma reesei using the pyrithiamine resistance gene ( $p \operatorname{tr} A)$ of Aspergillus oryzae. Biosci Biotechnol Biochem 2002, 66:404-406.

16. Schuster A, Kubicek CP, Friedl MA, Druzhinina IS, Schmoll M: Impact of light on Hypocrea jecorina and the multiple cellular roles of ENVOY in this process. BMC Genomics 2007, 8:449.

17. Guangtao Z, Seiboth B, Wen C, Yaohua Z, Xian L, Wang T: A novel carbon source dependent genetic transformation system for the versatile cell factory Hypocrea jecorina (anamorph Trichoderma reesei). FEMS Microbiol Lett 2010, 303:26-32.

18. Hartl L, Seiboth B: Sequential gene deletions in Hypocrea jecorina using a single blaster cassette. Curr Genet 2005, 48:204-211.

19. Steiger MG, Vitikainen M, Uskonen P, Brunner K, Adam G, Pakula T, Penttila M, Saloheimo M, Mach RL, Mach-Aigner AR: Transformation System for Hypocrea jecorina (Trichoderma reesei) That Favors Homologous Integration and Employs Reusable Bidirectionally Selectable Markers. Applied and environmental microbiology 2011, 77:114.

20. Guangtao Z, Hartl L, Schuster A, Polak S, Schmoll M, Wang T, Seidl V, Seiboth B: Gene targeting in a nonhomologous end joining deficient Hypocrea jecorina. J Biotechnol 2009, 139:146-151.

21. Critchlow SE, Bowater RP, Jackson SP: Mammalian DNA double-strand break repair protein XRCC4 interacts with DNA ligase IV. Curr Biol 1997, 7:588-598.

22. Hoff B, Kamerewerd J, Sigl C, Zadra I, Kück U: Homologous recombination in the antibiotic producer Penicillium chrysogenum: strain Pcku70 shows up-regulation of genes from the HOG pathway. Appl Microbiol Biotechnol 2010, 85:1081-1094.

23. Snoek I, van der Krogt Z, Touw H, Kerkman R, Pronk J, Bovenberg R, van den Berg M, Daran J: Construction of an hdfA Penicillium chrysogenum strain impaired in non-homologous end-joining and analysis of its potential for functional analysis studies. Fungal Genet Biol 2009, 46:418-426.

24. Kuhls K, Lieckfeldt E, Samuels GJ, Kovacs W, Meyer W, Petrini O, Gams W, Borner T, Kubicek CP: Molecular evidence that the asexual industrial fungus Trichoderma reesei is a clonal derivative of the ascomycete Hypocrea jecorina. Proc Natl Acad Sci USA 1996, 93:7755-7760.

25. Seidl V, Seibel C, Kubicek CP, Schmoll M: Sexual development in the industrial workhorse Trichoderma reesei. Proc Natl Acad Sci USA 2009, 106:13909-13914.

26. Wilson RA, Talbot NJ: Fungal physiology-a future perspective. Microbiology 2009, 155:3810.

27. Park G, Colot HV, Collopy PD, Krystofova S, Crew C, Ringelberg C Litvinkova L, Altamirano L, Li L, Curilla S: High-Throughput Production of Gene Replacement Mutants in Neurospora crassa. Methods Mol Biol 2011 722:179.

28. Tian C, Beeson W, lavarone A, Sun J, Marletta M, Cate J, Glass N: Systems analysis of plant cell wall degradation by the model filamentous fungus Neurospora crassa. Proc Natl Acad Sci USA 2009, 106:22157-22162.

29. Videira A, Kasuga T, Tian C, Lemos C, Castro A, Glass NL: Transcriptional analysis of the response of Neurospora crassa to phytosphingosine reveals links to mitochondrial function. Microbiology 2009, 155:3134

30. Colot HV, Park G, Turner GE, Ringelberg C, Crew CM, Litvinkova L, Weiss RL, Borkovich KA, Dunlap JC: A high-throughput gene knockout procedure for Neurospora reveals functions for multiple transcription factors. Proc Natl Acad Sci USA 2006, 103:10352-10357.

31. Tian C, Li J, Glass NL: Exploring the bZIP transcription factor regulatory network in Neurospora crassa. Microbiology 2011, 157:747.
32. Zeilinger S, Reithner B, Scala V, PeissI I, Lorito M, Mach RL: Signal transduction by Tga3, a novel G protein alpha subunit of Trichoderma atroviride. Applied and environmental microbiology 2005, 71:1591-1597.

33. Freitag M, Williams RL, Kothe GO, Selker EU: A cytosine methyltransferase homologue is essential for repeat-induced point mutation in Neurospora crassa. Proc Natl Acad Sci USA 2002, 99:8802.

34. Kays AM, Rowley PS, Baasiri RA, Borkovich KA: Regulation of conidiation and adenylyl cyclase levels by the Galpha protein GNA-3 in Neurospora crassa. Mol Cell Biol 2000, 20:7693-7705.

35. Vitikainen M, Arvas M, Pakula T, Oja M, Penttilä M, Saloheimo M: Array comparative genomic hybridization analysis of Trichoderma reesei strains with enhanced cellulase production properties. BMC Genomics 2010, 11:441.

36. McCluskey K, Wiest AE, Grigoriev IV, Lipzen A, Martin J, Schackwitz W, Baker SE: Rediscovery by whole genome sequencing: classical mutations and genome polymorphisms in Neurospora crassa. G3: Genes, Genomes, Genetics 2011, 1:303-316.

37. Bidard F, Benkhali JA, Coppin E, Imbeaud S, Grognet P, Delacroix H, Debuchy R: Genome-wide gene expression profiling of fertilization competent mycelium in opposite mating types in the heterothallic fungus Podospora anserina. PloS one 2011, 6:e21476.

38. Galagan JE, Selker EU: RIP: the evolutionary cost of genome defense. Trends Genet 2004, 20:417-423.

39. Le Crom S, Schackwitz W, Pennacchio L, Magnuson JK, Culley DE, Collett JR, Martin J, Druzhinina IS, Mathis H, Monot F: Tracking the roots of cellulase hyperproduction by the fungus Trichoderma reesei using massively parallel DNA sequencing. Proc Natl Acad Sci USA 2009, 106:16151.

40. Seidl V, Gamauf C, Druzhinina IS, Seiboth B, Hartl L, Kubicek CP: The Hypocrea jecorina (Trichoderma reesei) hypercellulolytic mutant RUT C 30 lacks a $85 \mathrm{~kb}$ (29 gene-encoding) region of the wild-type genome. BMC Genomics 2008, 9:327.

41. Diener SE, Chellappan MK, Mitchell TK, Dunn-Coleman N, Ward M, Dean RA Insight into Trichoderma reesei's genome content, organization and evolution revealed through BAC library characterization. Fungal Genet Biol 2004, 41:1077-1087.

42. Foreman PK, Brown D, Dankmeyer L, Dean R, Diener S, Dunn-Coleman NS, Goedegebuur F, Houfek TD, England GJ, Kelley AS: Transcriptional regulation of biomass-degrading enzymes in the filamentous fungus Trichoderma reesei. J Biol Chem 2003, 278:31988.

43. Arvas M, Haiminen N, Smit B, Rautio J, Vitikainen M, Wiebe M, Martinez D, Chee C, Kunkel J: Detecting novel genes with sparse arrays. Gene 2010, 467(1-2):41-51.

44. Portnoy T, Margeot A, Linke R, Atanasova L, Fekete E, Sandor E, Hartl L, Karaffa L, Druzhinina I, Seiboth B: The CRE1 carbon catabolite repressor of the fungus Trichoderma reesei: a master regulator of carbon assimilation. BMC Genomics 2011, 12:269.

45. Wilson DB: Cellulases and biofuels. Curr Opin Biotechnol 2009, 20:295-299.

46. Montenecourt B, Eveleigh D: Preparation of mutants of Trichoderma reesei with enhanced cellulase production. Applied and environmental microbiology 1977, 34:777-782.

47. Christianson TW, Sikorski RS, Dante M, Shero JH, Hieter P: Multifunctional yeast high-copy-number shuttle vectors. Gene 1992, 110:119-122.

48. Rozen S, Skaletsky H: Primer3 on the WWW for general users and for biologist programmers. Methods Mol Biol 2000, 132:365-386.

49. Collopy PD, Colot HV, Park G, Ringelberg C, Crew CM, Borkovich KA, Dunlap JC: High-throughput construction of gene deletion cassettes for generation of Neurospora crassa knockout strains. Methods Mol Biol 2010, 638:33.

50. Gietz RD, Schiestl RH: High-efficiency yeast transformation using the LiAc/ SS carrier DNA/PEG method. Nat Protoc 2007, 2:31-34

51. Schuster A, Kubicek CP, Schmoll M: The dehydrogenase GRD1 represents a novel component of the cellulase regulon in Trichoderma reesei (Hypocrea jecorina). Applied and environmental microbiology 2011, AEM.00513-00511.

doi:10.1186/1754-6834-5-1

Cite this article as: Schuster et al:: A versatile toolkit for high throughput functional genomics with Trichoderma reesei. Biotechnology for Biofuels 2012 5:1. 